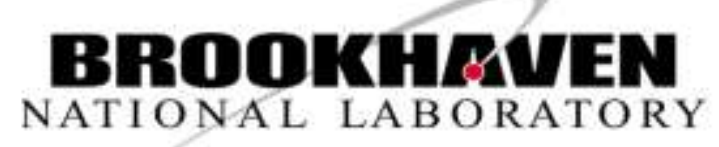

BNL-113996-2017-JA

\title{
High Indexed Pt3Ni Tetrahexahedral Nanoframes Evolved through Preferential CO Etching
}

\author{
C. Wang, L. Zhang, H. Yang, J. Pan, J. Liu, \\ C. Dotse, Y. Luan, R. Gao, C. Lin, J. Zhang, \\ J. P. Kilcrease, X. Wen, S. Zou and J. Fang
}

Submitted to Nano Lett.

April 2017

Center for Functional Nanomaterials

Brookhaven National Laboratory

\author{
U.S. Department of Energy \\ USDOE Office of Science (SC), \\ Basic Energy Sciences (SC-22)
}

Notice: This manuscript has been authored by employees of Brookhaven Science Associates, LLC under Contract No. DE-SC0012704 with the U.S. Department of Energy. The publisher by accepting the manuscript for publication acknowledges that the United States Government retains a non-exclusive, paid-up, irrevocable, world-wide license to publish or reproduce the published form of this manuscript, or allow others to do so, for United States Government purposes. 


\section{DISCLAIMER}

This report was prepared as an account of work sponsored by an agency of the United States Government. Neither the United States Government nor any agency thereof, nor any of their employees, nor any of their contractors, subcontractors, or their employees, makes any warranty, express or implied, or assumes any legal liability or responsibility for the accuracy, completeness, or any third party's use or the results of such use of any information, apparatus, product, or process disclosed, or represents that its use would not infringe privately owned rights. Reference herein to any specific commercial product, process, or service by trade name, trademark, manufacturer, or otherwise, does not necessarily constitute or imply its endorsement, recommendation, or favoring by the United States Government or any agency thereof or its contractors or subcontractors. The views and opinions of authors expressed herein do not necessarily state or reflect those of the United States Government or any agency thereof. 


\title{
High Indexed $\mathrm{Pt}_{3} \mathrm{Ni}$ Tetrahexahedral Nanoframes Evolved through Preferential CO Etching
}

\author{
Chenyu Wang, ${ }^{1 \dagger}$ Lihua Zhang, ${ }^{2 \dagger}$ Hongzhou Yang, ${ }^{3 \$}$ Jinfong Pan, ${ }^{4}$ Jingyue Liu, ${ }^{5}$ Charles Dotse, ${ }^{3}$ Yiliang \\ Luan, ${ }^{1}$ Rui Gao, ${ }^{6,7}$ Cuikun Lin, ${ }^{8}$ Jun Zhang, ${ }^{9}$ James P. Kilcrease, ${ }^{10}$ \\ Xiaodong Wen, ${ }^{6,7 *}$ Shouzhong Zou ${ }^{3,11^{*}}$ and Jiye Fang ${ }^{1,4^{*}}$
}

${ }^{1}$ Department of Chemistry, State University of New York at Binghamton, Binghamton, New York 13902, USA

${ }^{2}$ Center for Functional Nanomaterials, Brookhaven National Laboratory, Upton, New York 11973, USA

3Department of Chemistry and Biochemistry, Miami University, Oxford, Ohio 45056, USA

${ }^{4}$ Materials Science and Engineering Program, State University of New York at Binghamton, Binghamton, New York 13902, USA

${ }^{5}$ Department of Physics, Arizona State University, Tempe, Arizona 85287, USA

6Institute of Coal Chemistry, Chinese Academy of Sciences, Taiyuan, Shanxi 030001, China

${ }^{7}$ Synfuels China Co. Ltd., Huairou, Beijing 101407, China

8Department of Chemistry, University of South Dakota, Vermillion, South Dakota 57069, USA

${ }^{9}$ College of Chemical Engineering, China University of Petroleum, Qingdao, Shandong 266580, China

${ }^{10}$ Nanotechnology Systems Division, Hitachi High Technologies America, Inc., Clarksburg, Maryland 20871, USA

${ }^{11}$ Department of Chemistry, American University, Washington, District of Columbia 20016, USA

$\uparrow$ These authors contributed equally to this work.

\section{KEYWORDS:}

nanoframe, tetrahexahedron, high-index, preferential etching, $\mathrm{Pt}_{3} \mathrm{Ni}$ 


\begin{abstract}
:
Chemically controlling crystal structures in nanoscale is challenging, yet provides an effective way to improve catalytic performances. Pt-based nanoframes are a new class of nanomaterials that have great potential as high performance catalysts. To date these nanoframes are formed through acid etching in aqueous solutions, which demands long reaction time and often yields illdefined surface structures. Herein we demonstrate a robust and unprecedented protocol for facile development of high-performance nanoframe catalysts using size and crystallographic facet controlled $\mathrm{PtNi}_{4}$ tetrahexahedral nanocrystals prepared through a colloidal synthesis as precursors. This new protocol employs the Mond process to preferentially de-alloy nickel component in the $<100>$ direction through carbon monoxide etching of carbon supported $\mathrm{PtNi}_{4}$ tetrahexahedral nanocrystals at an elevated temperature. The resultant $\mathrm{Pt}_{3} \mathrm{Ni}$ tetrahexahedral nanoframes possess an open, stable and high-indexed microstructure, containing a segregated Pt thin layer strained to the Pt-Ni alloy surfaces, and featuring a down-shift $d$-band center as revealed by the density functional theory calculations. These nanoframes exhibit much improved catalytic performance, such as high stability under prolonged electrochemical potential cycles, promoting direct electro-oxidation of formic acid to carbon oxide and enhancing oxygen reduction reaction activities. Because carbon monoxide can be generated from the carbon support through thermal annealing in air, a common process for pre-treating supported catalysts, the developed approach can be easily adopted for preparing industrial scale catalysts that are made of Pt-Ni and other alloy nanoframes.
\end{abstract}


Platinum (Pt) is ubiquitously used in catalysis owing to its distinct surface electronic structure that enables its bonding to various adsorbates with appropriate strength. To maximize the utilization of this precious metal, extensive efforts have been geared to control their shape, size and composition of Pt and Pt-based alloy nanocrystals (NCs), which strongly correlate to the electronic structure and surface atomic arrangement. ${ }^{1}$ In particular, Pt-based alloy NCs have been extensively investigated due to the possibility to tune the $d$-band center position, which correlates to the adsorption strength of the catalyst surface, through the strain and ligand effects. ${ }^{2}$ Rationally engineering both the architecture and microstructure of Pt-based catalysts at nanoscale is an effective strategy to achieve desirable catalytic properties. ${ }^{3,4}$ De-alloying the less noble transition metal from Pt-bimetallic NCs is a common approach to produce porous architecture and microstructures with surface strains. ${ }^{5}$ These features have been proven as the key factors for enhancing catalytic activity. ${ }^{6}$ Electrochemical potential cycling and selective solution etching are frequently applied methods for de-alloying. However, the resultant nanostructures typically have severely deformed morphologies and defect-rich surfaces, which make it arduous to acquire pre-designed microstructure and overall architecture. ${ }^{5,7}$ In contrast, thermal annealing can relieve the excessive internal stress to avert plastic deformation. ${ }^{8}$ In addition, the thermal energy may drive surface restructuring to reach a thermodynamic equilibrium structure and thus generate a uniform microstructure where a $\mathrm{Pt}$ thin layer is segregated on the surface. In view of these lines of reasoning, de-alloying of Pt-based NCs through solid-state processing can be a more effective way to achieve 3D open architecture with controllable microstructures. ${ }^{9}$ Herein, we report a judiciously designed protocol for fabricating $\mathrm{Pt}_{3} \mathrm{Ni}$ tetrahexahedral (THH) nanoframes for $\mathrm{THH} \mathrm{PtNi}_{4} \mathrm{NCs}$ with controlled microstructures.

The THH shape was chosen in light of the improved catalytic activity of formic acid and ethanol electro-oxidation on Pt THH NCs with high indexed facets. ${ }^{10} \mathrm{PtNi}_{4} \mathrm{THH}$ NCs with welldefined $\{730\}$ facets and segregated Pt on the edges were synthesized as the "precursors" via a colloidal method as detailed in Supporting Information as well as in Fig. 1a. ${ }^{11}$ An unprecedented process to de-alloy and extract $\mathrm{Ni}$ from $\mathrm{PtNi}_{4}$ THH NCs was carried out by annealing carbonsupported $\mathrm{PtNi}_{4} \mathrm{NCs}$ in the presence of carbon monoxide (CO) through the Mond process (refer to Supporting Information). ${ }^{12}$ The obtained $\mathrm{Pt}_{3} \mathrm{Ni}$ nanoframes preserve the THH profile and present 3D open-structure comprising ridges as thin as a few nanometers (Figs. $1 \mathrm{~b}$ and 1c). In 
this process, the selective etching of $\mathrm{Ni}$ atoms from the $<100>$ direction is considered as a critical factor that prevents the deformation of THH frames, whereas the internal stress generated during the hollowing process is relieved through the thermal annealing and the accompanied surface restructuring. Moreover, the thermal treatment resulted in an alloy of the segregated Pt and the remaining Ni atoms, diminishing the uncontrollable vacancy defects and generating stable and well-defined microstructures of a strained $\mathrm{Pt}$ thin layer on top of $\mathrm{Pt}_{3} \mathrm{Ni}$ high-indexed planes. The resultant $\mathrm{Pt}_{3} \mathrm{Ni} \mathrm{THH}$ nanoframes are robust, and remain intact even after 5000 electrochemical potential cycles in $\mathrm{HClO}_{4}$ (Fig. 1d). Such $\mathrm{Pt}_{3} \mathrm{Ni} \mathrm{THH}$ nanoframes not only provide easy access for reactants to both internal and external active sites, but also possess desirable electronic structure for the adsorption of molecules, rendering superior catalytic performance. This method provides an ideal paradigm for engineering both the architecture and the microstructure of NCs through a combination of colloidal approach and solid-state processing, which may be applied to other bimetallic systems.
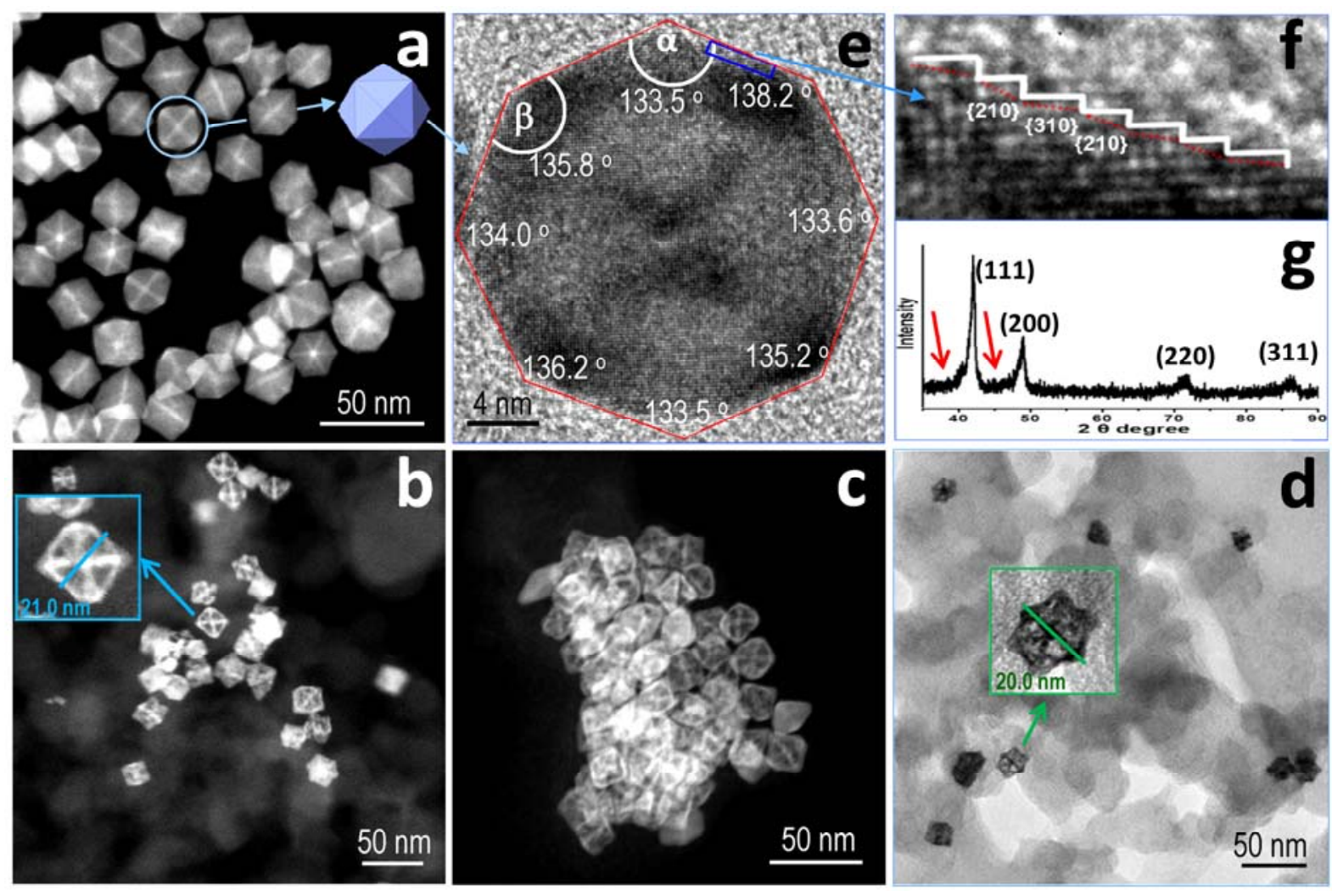

Figure 1. EM images of THH NCs and nanoframes. a, HAADF-STEM image of THH $\mathrm{PtNi}_{4} \mathrm{NCs}$ (the inset is a THH model along the [001] zone); $b$, HAADF-STEM image of THH nanoframes on carbon evolved by CO thermal annealing; c, HAADF-STEM image of THH nanoframes on carbon yielded by annealing in air; $d$, TEM image of air-annealing generated nanoframes after 5000 electrochemical potential cycles from 0.6 
to $1.0 \mathrm{~V}$ vs RHE at $0.5 \mathrm{~V} / \mathrm{s}$ in $0.1 \mathrm{M} \mathrm{HClO}_{4}$, showing that the THH profile is perfectly preserved; e, HRTEM of a single $\mathrm{PtNi}_{4} \mathrm{THH}$ NC recorded along the [001] zone axis with an indexation of the measured angles between surfaces; f, HRTEM image zoomed from the boxed area in (e), displaying the surface atomic steps marked as $\{210\}$ and $\{310\}$; a corresponding FFT pattern with a square symmetry illustrated in Fig. S1D confirms the [001] projection orientation; g, XRD pattern of the obtained THH NCs, showing the alloy phase with the $f c c$ structure.

Well-defined Pt-Ni polyhedral NCs exhibit outstanding electro-catalytic activities, and the tendency to form segregated structures which are ideal templates for generating nanoframes. ${ }^{6}$ In this work, Pt-Ni NCs with THH morphology, which are terminated with 24 isosceles triangular high-indexed facets as illustrated in Fig. S1a, were selected as the "precursors". We developed a robust synthesis approach ${ }^{11}$ (see supplementary materials for the details) to harvest sizecontrolled $\mathrm{PtNi}_{4}$ THH NCs. The obtained Pt-Ni NCs feature a segregated atomic distribution, in which Pt atoms are concentrated at the edges and corners while Ni atoms are centralized in the interior, forming "Pt-rich frameworks" filled with Ni. A high-angle annular dark-field scanning transmission electron microscopic (HAADF-STEM) image (Fig. 1a) and a bright-field transmission electron microscopic (TEM) image (Fig. S1b) show an average size of $\sim 22 \mathrm{~nm}$ in projection "diameter" of the as-synthesized THH NCs. As discerned from three representative NCs circled in the HAADF-STEM image (Fig. S1c), the projection profiles of these THH NCs closely resemble those of ideal THH models with different orientations. A high-resolution TEM (HRTEM) image of a single Pt-Ni THH NC was recorded along the [001] zone axis (Fig. 1e), and this projection orientation is confirmed by the corresponding Fourier transform (FT) pattern of a square symmetry (Fig. S1d). The average values of projection angles, $\alpha$ and $\beta$, are determined as $133.6(5)^{\circ}$ and $136.3(5)^{\circ}$ from a series of measurements marked on the HRTEM image (Fig. 1f). According to the projection angle formula ${ }^{13} \alpha=2 \arctan \left(\frac{h}{k}\right)$, the average value of $\mathrm{h} / \mathrm{k}$ can be calculated as $2.3360 \pm 0.0135 \approx 7 / 3$ and the miller indices of the surface planes are therefore deduced as $\{730\}^{10,14}$ (also refer to the theoretical angles in Fig. S2). The indexation of the exposed facets can be corroborated from the atomic arrangement, in which $\{310\}$ and $\{210\}$ sub-facets periodically stack up, as delineated in Fig. 1f. It should be noted that the edges of the THH NCs present different Z-contrast compared with the interior (Fig. 1a and Fig. S1c), inferring a non-uniform compositional distribution from the edges to the center as reported before. $^{15}$ This characteristic is also reflected in the X-ray diffraction (XRD) pattern (Fig. 1g). The aslant peak shoulders marked by the red arrows suggest the underlying segregation of 


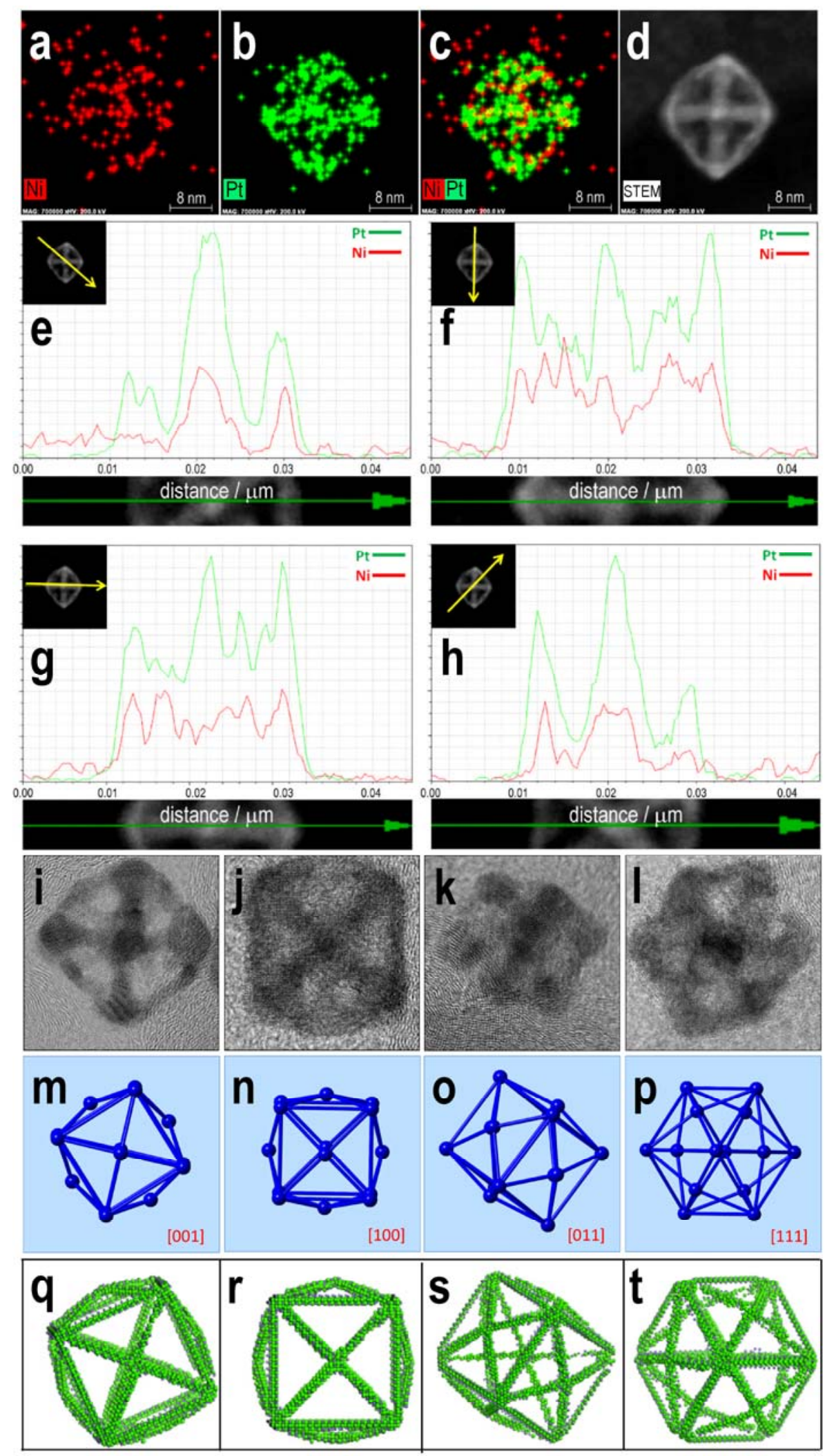

Figure 2. Elemental mapping, HAADF-STEM images and EDX line scan profiles of $\mathrm{Pt}_{3} \mathrm{Ni} \mathrm{THH}$ nanoframes, yielded via CO-annealing at $170{ }^{\circ} \mathrm{C}$ for 45 minutes. a-c, HAADF-STEM EDX elemental mapping, d, HAADF- 
STEM image on a selected THH nanoframe in the zone axis of [001], showing its Pt-rich hollow structure and Ni removal. e-h, HAADF-STEM EDX line scan profiles on the THH nanoframe along the [001] zone axis with four different scan paths. $\mathrm{i}-\mathrm{I}$, HRTEM images of THH nanoframes in different zone axes as illustrated in models of $(m-p)$ and $(q-t)$, respectively. In (q-t), the pewter and green colors represent Pt and $\mathrm{Pt}_{3} \mathrm{Ni}$ (not to the atomic ratio), respectively.

components (as opposed to a core-shell structure), ${ }^{16}$ which agrees with a recent report. ${ }^{17}$ Energydispersive X-ray spectroscopy (EDX) analysis, as presented in Figs. S3-S4, confirms that in a THH NC more Pt atoms are distributed at the edge and corners, whereas more Ni atoms are segregated in the center. The global $\mathrm{Pt} / \mathrm{Ni}$ atomic ratio is 1:4 as assessed by inductively coupled plasma-optical emission spectrometry (ICP-OES) (Table S1).

The $\mathrm{PtNi}_{4} \mathrm{THH}$ NCs can be transformed into $\mathrm{Pt}_{3} \mathrm{Ni} \mathrm{THH}$ nanoframes by selectively removing $\mathrm{Ni}$ through the Mond process, i.e. thermal annealing under CO atmosphere. ${ }^{12,18}$ Figs. 2a-2d shows HAADF-STEM EDX elemental mapping and imaging on a CO-etched nanoframe in the zone axis of [001], presenting a Pt-rich hollow structure with the THH profile. Abundance of Pt (green) in the proximity of rims and corners can be clearly seen in Figs. 2b and 2c. The detection of $\mathrm{Ni}$ signals outside the nanoframes indicates the extraction of $\mathrm{Ni}$ is through the formation and removal of the volatile $\mathrm{Ni}(\mathrm{CO})_{4}$ complexes. Pt-rich rims and apexes can be further verified by HAADF-STEM EDX line scan profiles obtained alone the [001] zone axis with four different scan paths as illustrated in Figs. 2e-2h. Compositional analyses on these THH nanoframes (also refer to Fig. S5) from different orientations (Figs. 2i-2t) are presented in Fig. S6. It is revealed that the CO-etching time controls the extent of de-alloying and the EDX data acquired at different positions along the [001] zone axis on a selected THH nanoframe etched for 45 minutes show an average $\mathrm{Pt} / \mathrm{Ni}$ molar ratio of $\sim 3: 1$ (Table $\mathrm{S} 2$ ).

Unlike most of the etched NCs with deformed morphologies, the overall THH profile and exposed high-indexed facets in our Pt-Ni NCs are retained despite that significant amounts of $\mathrm{Ni}$ are removed. As shown in Fig. $3 b$ and Fig. S7, a series of $\{h k 0\}$ facets including $\{510\}$ as well as $\{310\},\{410\}$ and $\{720\}$ can be clearly identified, indicating the presence of high-indexed facets in these THH nanoframes. It should be noted that the surface of these hollow nanoarchitectures are quite smooth, distinctly different from those rough surfaces with dangling bonds produced via solution etching. Such a feature is essential to maintaining the surface 
stability and averting further Ni leaching in the acidic/corrosive catalytic environment. Close inspection of the outmost (200) lattice (segregated with Pt) of a solid THH NC (Fig. 3a) and a THH nanoframe (Fig. 3b) reveals a shrinkage in lattice spacing, from $1.95 \AA$ on the solid THH NC (Fig. 4c) to $1.92 \AA$ on the nanoframe (Fig. 3d). Both values are slightly less than that of $\mathrm{Pt}(200)$, suggesting that the segregated Pt thin shell is strained to the Pt-Ni alloy core. This compressed strain is consistent with the results predicted by density function theory (DFT) calculations (2.01 $\AA$ vs. $1.93 \AA$ ). The difference in lattice contraction between solid and hollow THH NCs likely arises from the decrease of coordinating atoms for those segregated Pt atoms on Pt-Ni nanoframes due to the loss of Ni. Such compressive strains would result in a down-shift of the $d$-band center (relative to the Fermi level) ${ }^{19}$ and potentially render these NCs unique catalytic properties by changing the surface binding strength.

Generally, annealing would result in the formation of thermodynamically stable structure. Why then in this case the high indexed facets are preserved? To monitor the "etching" process, controlled experiments were conducted by shortening the annealing time. The NC intermediates with shallow voids on the surfaces were observed. Comparison of an intermediate (Fig. 3e-3h) with an original THH NC (Fig. 3i-31) shows that a cuboid Ni profile was left behind in the center after the surface $\mathrm{Ni}$ atoms were etched away, which suggests preferential removal of $\mathrm{Ni}$ from the $<100>$ direction. For the rims and edges where most Pt are found, the preservation of highindexed facets can be attributed to the preferential segregation of $\mathrm{Ni}$ on the outmost layer of the Pt-Ni alloys in certain orientations. ${ }^{20,21}$ The bulk $\mathrm{Ni}$ atoms are driven to the top layer continuously until a more stable hierarchical microstructure with a thin layer of Pt on top of the Pt-Ni high-indexed frames is formed. Meanwhile, the mild annealing temperature $\left(<200{ }^{\circ} \mathrm{C}\right)$ also helps to release the stress caused by the extraction of $\mathrm{Ni}$.

For electrocatalytic applications, metal NCs are frequently supported on high surface area carbon blacks to help disburse the particles. It is known from the fuel cell research that the carbon support can be oxidized to release $\mathrm{CO}$ and $\mathrm{CO}_{2}$ at elevated temperatures especially in the presence of $\mathrm{Pt}^{22,23}$ We further explore the possibility of using $\mathrm{CO}$ generated from the partial oxidation of the carbon support to etch away the $\mathrm{Ni}$ from $\mathrm{PtNi}_{4} \mathrm{THH}$ NCs in one-step annealing. We annealed the carbon supported solid $\mathrm{PtNi}_{4} \mathrm{THH} \mathrm{NCs}$ at $185{ }^{\circ} \mathrm{C}$ in air followed by further annealing at $300{ }^{\circ} \mathrm{C}$ in $4 \% \mathrm{H}_{2} / \mathrm{N}_{2}$ (denoted as "air-annealing process", refer to "materials and 
methods" in see supplementary materials). The second annealing step is to ensure the reduction of metal oxides that may be generated in this process. Supported metal catalysts commonly undergo a similar thermal annealing procedure. ${ }^{3,24}$ However, in the present case the carbon black serves as both the support and the CO source. Most of the NCs after the "air-annealing process" show same features as those from nanoframes evolved under pure CO annealing, including open structure, preservation of THH profiles, and the presence of lattice strain (refer to Figs. S8-S9 and related discussion). The etching mechanism in the "air-annealing" process is the same as that annealed in pure $\mathrm{CO}$, as confirmed by the detection of $\mathrm{Ni}$ on the carbon support (Figs. S10 and S11).

These hollow nanostructures with well-defined surface planes and engineered microstructures are ideal for studying the structure-property dependence. The $\mathrm{Pt}_{3} \mathrm{Ni} \mathrm{THH}$ nanoframes show superior catalytic activity to the commercial Pt/C (HiSpec 4000, $40 \mathrm{wt} \% \mathrm{Pt}$ ). As shown in the cyclic voltammograms (CVs) in Fig. $4 \mathrm{a}$, on $\mathrm{Pt}_{3} \mathrm{Ni} \mathrm{THH}$ nanoframes the formic acid oxidation current density (normalized to Pt area) in the anodic scan (from negative to positive potentials) is very close to the cathodic scan, in stark contrast to $\mathrm{Pt} / \mathrm{C}$ where the anodic scan current density is much lower than the cathodic scan. At $0.40 \mathrm{~V}$, the cathodic to anodic scan current ratio is 1.7 on $\mathrm{Pt}_{3} \mathrm{Ni} \mathrm{THH}$ nanoframes, but 9.1 on $\mathrm{Pt} / \mathrm{C}$, suggesting that the $\mathrm{Pt}_{3} \mathrm{Ni} \mathrm{THH}$ nanoframe is much less vulnerable to surface poisoning. This conclusion is confirmed by the transient current density recorded at $0.30 \mathrm{~V}$ vs RHE in Fig. 4b. The current density on $\mathrm{Pt}_{3} \mathrm{Ni}$ THH nanoframe is much higher than $\mathrm{Pt} / \mathrm{C}$ in the entire time. For example, at $60 \mathrm{~s}$, the activity of $\mathrm{Pt}_{3} \mathrm{Ni}$ THH nanoframe is 4 times of $\mathrm{Pt} / \mathrm{C}$. 

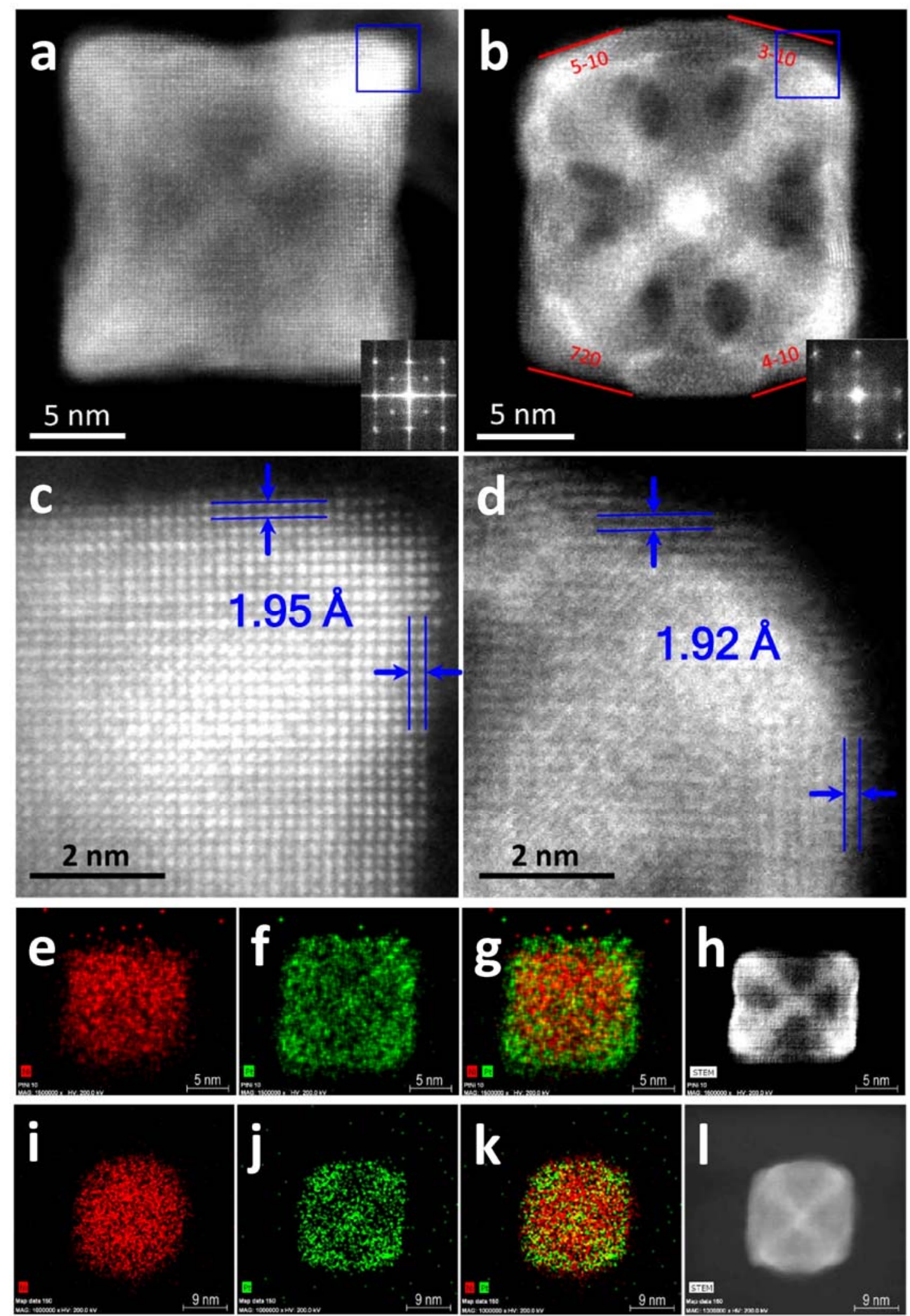

Figure 3. HAADF-STEM images and EDX elemental mapping results in zone axis of the [100]. a-d, HAADFSTEM images of a THH nanoframe generated from CO-etching at $170{ }^{\circ} \mathrm{C}$ for 45 minutes. a, THH NC; $b$, $\mathrm{THH}$ nanoframe yielded via CO-etching. Insets in (a) and (b) are FFT patterns of their whole images, 
respectively, confirming the [100] projection orientation. $c$ and d are zoom-in areas illustrated in (a) and (b), respectively, with average results of the measured (200) lattice fringes. e-l, comparative HAADFSTEM EDX elemental mapping results in the zone axis of [001]. e-g and i-k, HAADF-STEM EDX elemental mapping images; $h$ and I, HAADF-STEM images of NCs; e- $h$, nanoframe resulted from a partial COetching process; $\mathrm{i}-\mathrm{I}, \mathrm{PtNi}_{4}$ THH NC (before the Ni-extraction).

To gain insights into the significantly enhanced formic acid oxidation activity on $\mathrm{Pt}_{3} \mathrm{Ni} \mathrm{THH}$ nanoframes, we calculated the energetics of reaction pathways using DFT. The Pt-skin layer on top of $\mathrm{Pt}_{3} \mathrm{Ni}(730)$ was taken into account in the DFT calculations by including 2 to $3 \mathrm{Pt}$ top layers denoted as $\mathrm{Pt} / \mathrm{Pt}_{3} \mathrm{Ni}(730)$. Although the nanoframes also contain other surfaces, such as (310) and (210), the energetics on these surfaces are similar to the $\mathrm{Pt} / \mathrm{Pt}_{3} \mathrm{Ni}(730)$.
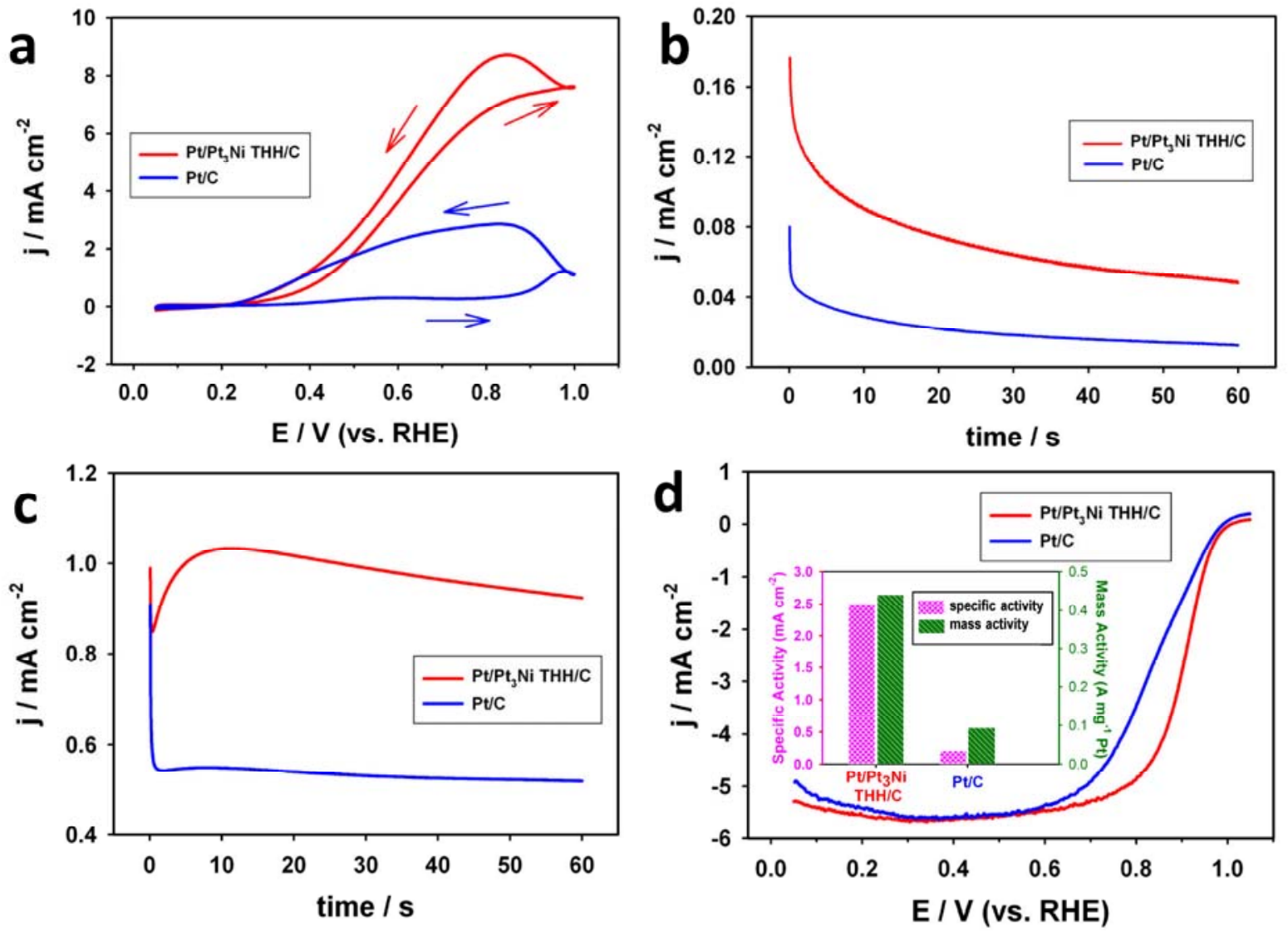

Figure 4. Comparison of electrocatalytic activity per unit Pt surface area between $\mathrm{Pt}_{3} \mathrm{Ni} \mathrm{THH} / \mathrm{C}$ and $\mathrm{Pt} / \mathrm{C}$. a, CVs in $0.1 \mathrm{M} \mathrm{HClO}_{4}+0.5 \mathrm{M} \mathrm{HCOOH}$ at a scan rate of $0.1 \mathrm{~V} \mathrm{~s}^{-1} ; b$, Current density-time curves recorded in the same solution at $0.30 \mathrm{~V}$ (vs. RHE); c, Current density-time curves recorded in $0.1 \mathrm{M} \mathrm{HClO}_{4}+1 \mathrm{M}$ $\mathrm{CH}_{3} \mathrm{OH}$ at $0.75 \mathrm{~V}$ (vs RHE); d, ORR polarization curves recorded in $\mathrm{O}_{2}$-saturated $0.1 \mathrm{M} \mathrm{HClO}_{4}$ with a potential scan rate of $20 \mathrm{mV} \mathrm{s}^{-1}$ and an electrode rotation rate of $1600 \mathrm{rpm}$; Insert of (d): ORR activity comparison at $0.90 \mathrm{~V}$ vs RHE. 
It is well known that the electro-oxidation of formic acid to $\mathrm{CO}_{2}$ proceeds through the socalled dual-path reaction mechanism: a "direct" route involving two dehydrogenation steps of formic acid to $\mathrm{CO}_{2}$ and an "indirect" way associated with the formation of $\mathrm{CO}_{\mathrm{ad}}$ acting as a surface blocking species at potentials relevant to fuel cell applications. ${ }^{25,26}$ The calculation results (Fig. S12) show that on $\mathrm{Pt} / \mathrm{Pt}_{3} \mathrm{Ni}(730)$ the barriers for dehydrogenation to form $\mathrm{CO}_{2}$ and $\mathrm{H}_{2}$ are much lower than on $\mathrm{Pt}(111)$ which corroborate with the measured higher activity on $\mathrm{Pt}_{3} \mathrm{Ni}$ THH nanoframes. Moreover, the decomposition of $\mathrm{COOH}^{*}$ to $\mathrm{CO}^{*}$ and $\mathrm{OH}^{*}$ on $\mathrm{Pt}_{3} \mathrm{Ni}(730)$ has a barrier of $1.38 \mathrm{eV}$, much higher than $0.71 \mathrm{eV}$ found on $\mathrm{Pt}(111)$, indicating the nanoframes are much more resistant to the surface poisoning by adsorbed $\mathrm{CO}$, which agrees well with the experimental results presented in Fig. 4. The DFT calculations also reveal the catalytic significance of the formation of $\mathrm{Pt}$ top layers on $\mathrm{Pt}_{3} \mathrm{Ni}$ THH nanoframes. As revealed in Fig. S12, the activation energy for dehydrogenation of formic acid through the direct pathway on $\mathrm{Pt} / \mathrm{Pt}_{3} \mathrm{Ni}(730)$ is $0.26 \mathrm{eV}$, significantly lower than $0.38 \mathrm{eV}$ detected on $\mathrm{Pt}_{3} \mathrm{Ni}(730)$, which represents pristine $\mathrm{Pt}_{3} \mathrm{Ni}$ THH NCs. The barrier for $\mathrm{COOH}$ dehydrogenation on the $\mathrm{Pt}_{3} \mathrm{Ni}(730)$ surface is essentially identical to that on $\operatorname{Pt}(111)(0.41 \mathrm{eV})$.

The improved formic acid oxidation activity may be attributed to the less binding strength to reactants on the Pt-skin layer. The Pt-Pt distance is about 2\% smaller than the pure Pt. This compressive strain has been shown to decrease the Pt $d$-band center and therefore weaken the adsorption of species on the surface. ${ }^{27,28}$ The effect of the compressive strain on the catalytic activity is further manifested in other reactions of significance to fuel cell applications. The onset potential of $\mathrm{CO}$ electro-oxidation on $\mathrm{Pt}_{3} \mathrm{Ni}$ THH nanoframes is about $30 \mathrm{mV}$ more negative than on $\mathrm{Pt} / \mathrm{C}$ (Fig. S13). The enhanced CO oxidation contributes to the $180 \%$ improvement of methanol oxidation observed on $\mathrm{Pt}_{3} \mathrm{Ni}$ THH nanoframes over Pt/C at $60 \mathrm{~s}$ (Fig. 4c). It has been well documented on other PtNi systems that the compressive strain lowers the oxygen binding energy and increases the oxygen reduction reaction (ORR) activity. ${ }^{6,29,30} \mathrm{The} \mathrm{Pt}_{3} \mathrm{Ni} \mathrm{THH}$ nanoframes also exhibit significantly improved ORR activity: the specific activity normalized to the Pt surface area is more than 10 times higher than the $\mathrm{Pt} / \mathrm{C}$ and the mass activity shows a nearly 5 time improvement (Fig. 4d).

The $\mathrm{Pt}_{3} \mathrm{Ni}$ THH/C nanoframes are stable in catalytic environments. Fig. S14 shows HAADFSTEM images and HAADF-STEM EDX elemental mapping on several nanoframes that were 
collected after 5000 potential cycles between 0.6 and $1.0 \mathrm{~V}$, demonstrating no apparent changes on both the morphology and composition. Based on an average of 50 point spectra acquired at different positions of several particles, a local Pt/Ni molar ratio of 3:1 (73.4 / 26.6 $\approx 3: 1)$ was confirmed, demonstrating the high stability of such THH nanoframes in the acidic electrochemical environment. The highly stable nanoframe structures after the 5000 electrochemical potential cycles are further displayed in the two TEM videos attached in the supplementary materials.

\section{CONCLUSIONS:}

We have presented a facile and robust approach to generate high-indexed $\mathrm{Pt}_{3} \mathrm{Ni}$ THH nanoframes via carbon monoxide etching at elevated temperatures. The nanoframes feature welldefined high indexed exposed surfaces, whose formation is attributed to the preferential etching pathway and mild annealing temperature. Unlike solution-based etching, this developed protocol shortens the etching time and generates well-defined surface structures. The segregated compressively strained Pt thin layer on the outmost of rims and vertexes has a downshifted $d$ band center which is mainly responsible for the improved catalytic performance. The obtained nanoframes address some of the major challenges for advanced catalysts with high stability and activity. In addition, through the same reaction the nanoframes can be formed by annealing the carbon black supported precursor in air, a common practice for preparing industrial nanocatalysts, which offers straightforward incorporation of the developed approach in industry to engineer surface-structure controlled catalysts. It is foreseeable that this approach can be extended to other bimetallic systems for fabricating a variety of desirable and stable microstructures.

\section{ASSOCIATED CONTENT:}

\section{Supporting Information:}

The Supporting Information is available free of charge on the ACS Publications website at DOI: xxx. Materials, Synthesis, Characterization and Computation Methods, Results and Discussion (Figs. S1-S14 and discussion; Fig. S15 (videos); Tables S1 and S2, and Reference list (PDF). 


\section{AUTHORS' INFORMATION:}

Corresponding Authors:

Jiye Fang: jfang@binghamton.edu; Shouzhong Zou: szou@american.edu; Xiaodong Wen: wxd@sxicc.ac.cn

Present Addresses:

Hongzhou Yang: Present address: Dioxide Materials, Inc., 3998 FAU Boulevard \#300, Boca Raton, FL 33431

Notes:

The authors declare no competing financial interest.

\section{ACKNOWLEDGEMENTS:}

This work is partially supported by Custom Electronics Incorporated to JF and NSF (CHE1156425, CHE-1559670) to SZ. J.L. acknowledges the support by Arizona State University, NSF under CHE-1465057, and the use of facilities in the John M. Cowley Center for High Resolution Electron Microscopy at Arizona State University. X.W. thanks the National Thousand Young Talents Program of China, Hundred-Talent Program of Chinese Academy of Sciences and Shanxi Hundred-Talent Program. The computational resources were provided by the Tianhe-2 in Lvliang, Shanxi and National Supercomputing Center in Shenzhen. This research utilized Hitachi2700C STEM and JEOL2100F HRTEM at the Center for Functional Nanomaterials, which is supported by U.S. DOE Office of Science Facility, at Brookhaven National Laboratory under Contract No. DE-SC001270. The authors thank Dr. Pengju Ren for useful computation assistance.

\section{REFERENCES AND NOTES:}

(1) Chen, J.; Lim, B.; Lee, E. P.; Xia, Y. NanoToday 2009, 4, 81-95.

(2) Kitchin, J. R.; Nørskov, J. K.; Barteau, M. A.; Chen, J. Phys. Rev. Lett. 2004, 93, 156801.

(3) Kang, Y.; Qi, L.; Li, M.; Diaz, R. E.; Su, D.; Adzic, R. R.; Stach, E.; Li, J.; Murray, C. B. ACS Nano 2012, 6, 2818-2825.

(4) Zhang, L.; Roling, L. T.; Wang, X.; Vara, M.; Chi, M.; Liu, J.; Choi, S.-I.; Park, J.; Herron, J. A.; Xie, Z.; Mavrikakis, M.; Xia, Y. Science 2015, 349, 412-416.

(5) Strasser, P.; Koh, S.; Anniyev, T.; Greeley, J.; More, K.; Yu, C.; Liu, Z.; Kaya, S.; Nordlund, D.; Ogasawara, H. Nat. Chem. 2010, 2, 454-460. 
(6) Chen, C.; Kang, Y.; Huo, Z.; Zhu, Z.; Huang, W.; Xin, H. L.; Snyder, J. D.; Li, D.; Herron, J. A.; Mavrikakis, M.; Chi, M.; More, K. L.; Li, Y.; Markovic, N. M.; Somorjai, G. A.; Yang, P.; Stamenkovic, V. R. Science 2014, 343, 1339-1343.

(7) Wu, Y.; Wang, D.; Chen, X.; Zhou, G.; Yu, R.; Li, Y. J. Am. Chem. Soc. 2013, 135, 12220-12223.

(8) Callister, W. D.; Rethwisch, D. G. Materials Science and Engineering: An Introduction; 8th ed.; John Wiley \& Sons: NJ, 2007; Vol. 7.

(9) Ringe, E.; Van Duyne, R.; Marks, L. Nano Lett. 2011, 11, 3399-3403.

(10) Tian, N.; Zhou, Z.-Y.; Sun, S.-G.; Ding, Y.; Wang, Z. L. Science 2007, 316, 732-735.

(11) Pan, J., PhD Desertation: "Evolution of nanocrystals in solution phase: synethsis, shape control and growth studies", State University of New York at Binghamton, 2013.

(12) Roberts-Austen, W. C. Nature 1898, 59, 63-64.

(13) Quan, Z.; Wang, Y.; Fang, J. Acc. Che,. Res. 2013, 46, 191-202.

(14) Zhou, Y.; Zhang, J.; Su, G.; Li, J. Sci. Rep. 2014, 4, 6520.

(15) Gan, L.; Cui, C.; Heggen, M.; Dionigi, F.; Rudi, S.; Strasser, P. Science 2014, 346, 15021506.

(16) Cui, C.; Gan, L.; Heggen, M.; Rudi, S.; Strasser, P. Nat. Mater. 2013, 12, 765-771.

(17) Ding, J.; Bu, L.; Guo, S.; Zhao, Z.; Zhu, E.; Huang, Y.; Huang, X. Nano Lett. 2016, 16, 2762-2767.

(18) Ludwig Mond ever demonstrated that element Ni could be extracted from its ores above $50{ }^{\circ} \mathrm{C}$ by $\mathrm{CO}$ that acts as both the complex ligand and reducing agent to form a gaseous compound, $\mathrm{Ni}(\mathrm{CO})_{4}$, which is stable up to $230{ }^{\circ} \mathrm{C}$. Above $230{ }^{\circ} \mathrm{C}$, $\mathrm{Ni}$ and $\mathrm{CO}$ can be recovered again from the decomposition $\mathrm{Ni}(\mathrm{CO})_{4}$.

(19) Calculated d-band center values on the three surfaces: $\mathrm{Pt}(111),-1.707 \mathrm{eV} ; \mathrm{Pt}_{3} \mathrm{Ni}(730)$, $1.985 \mathrm{eV}$; and $\mathrm{Pt} / \mathrm{Pt}_{3} \mathrm{Ni}(730),-1.992 \mathrm{eV}$. All calculations were carried out using Vienna ab initio Simulation Package (VASP) based on the projector-augmented wave pseudo-potential.

(20) Weigand, P.; Jelinek, B.; Hofer, W.; Varga, P. Surf. Sci. 1994, 301, 306-312.

(21) Hofer, W. Fresenius J. Anal. Chem. 1993, 346, 246-251.

(22) Stevens, D. A.; Dahn, J. R. Carbon 2015, 43, 179-188.

(23) Baturina, O. A.; Aubuchon, S. R.; Wynne, K. J. Chem. Mater. 2006, 18, 1498-1504.

(24) Srivastava, R.; Mani, P.; Hahn, N.; Strasser, P. Angew. Chem. Int. Ed. 2007, 46, 89888991.

(25) Marković, N.; Ross Jr, P. Surdf. Sci. Rep. 2002, 45, 117-229.

(26) Parsons, R.; VanderNoot, T. J. Electroanal. Chem. Interf. Electrochem. 1988, 257, 9-45.

(27) Yang, H.; Zhang, J.; Sun, K.; Zou, S.; Fang, J. Angew. Chem. Int. Ed. 2010, 49, 68486851. 
(28) Stamenkovic, V. R.; Mun, B. S.; Arenz, M.; Mayrhofer, K. J.; Lucas, C. A.; Wang, G.; Ross, P. N.; Markovic, N. M. Nat. Mater. 2007, 6, 241-247.

(29) Choi, S.-I.; Xie, S.; Shao, M.; Odell, J. H.; Lu, N.; Peng, H.-C.; Protsailo, L.; Guerrero, S.; Park, J.; Xia, X.; Wang, J.; Kim, M. J.; Xia, Y. Nano Lett. 2013, 13, 3420-3425.

(30) Zhang, J.; Yang, H.; Fang, J.; Zou, S. Nano. Lett. 2010, 10, 638-644.

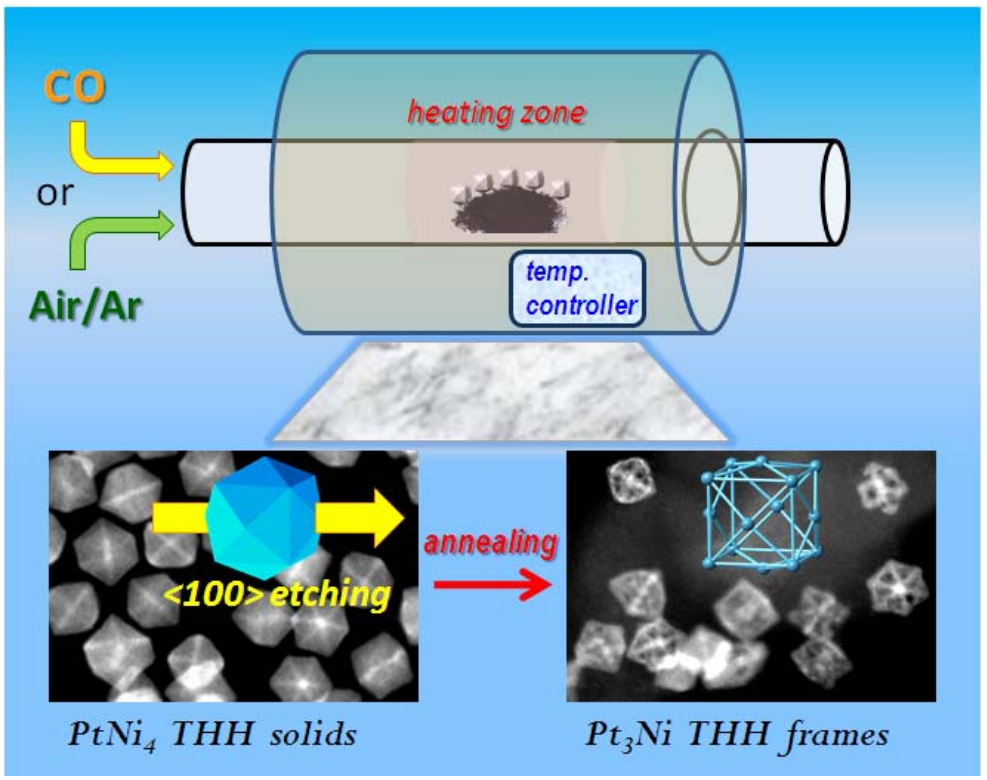

TOC. A schematic illustration of Mond process-based gas-etching protocol for $\mathrm{Pt}_{3} \mathrm{Ni}$ tetrahexahedroal nanoframe fabrication. 